\title{
Screening and identification of post-traumatic stress-related serum factors in children with Wilms' tumors
}

\author{
JUNJIE ZHANG ${ }^{1}$, QIAN HU ${ }^{2}$, FEI GUO ${ }^{1}$, LEI WANG ${ }^{1}$, WEI ZHAO ${ }^{1}$, DA ZHANG $^{1}$, HEYING YANG ${ }^{1}$, \\ JIEKAI YU ${ }^{3}$, LILI NIU ${ }^{4}$, FUQUAN YANG ${ }^{4}$, SHU ZHENG $^{3}$ and JIAXIANG WANG ${ }^{1}$ \\ ${ }^{1}$ Department of Surgery, The First Affiliated Hospital, Zhengzhou University; \\ ${ }^{2}$ Department of Nursing, Zhengzhou Railway Vocational and Technical College, Zhengzhou, Henan 450052; \\ ${ }^{3}$ Institute of Cancer, The Second Affiliated Hospital, College of Medicine, Zhejiang University, Hangzhou, Zhejiang 310000; \\ ${ }^{4}$ Proteomic Platform, Institute of Biophysics, Chinese Academy of Sciences, Beijing 100000, P.R. China
}

Received June 1, 2014; Accepted June 16, 2015

DOI: $10.3892 / 01.2016 .4099$

\begin{abstract}
Wilms' tumors are one of the most common malignant, solid intra-abdominal tumors observed in children. Although potential tumor markers have been found, inflammatory cytokines interfere with the process of specific protein identification. The present study was undertaken to identify post-traumatic stress-related factors of Wilms' tumors and to verify the accuracy of early-stage tumor-specific serum protein markers. Serum samples were screened for differentially-expressed proteins using surface-enhanced laser desorption/ionization-time of flight mass spectrometry (SELDI-TOF-MS). Potential markers were isolated and purified using solid-phase extraction (SPE) and SDS-PAGE. Following enzymatic digestion of the protein samples, the peptide fragments were detected with high performance liquid chromatography-mass spectrometry. The obtained peptide mass fingerprint was searched in the Swiss-Prot protein sequence database via the Mascot search engine. Differentially-expressed proteins were verified using western blot analysis. Differentially-expressed proteins with a mass/charge of 5,816 were screened out using SELDI-TOF-MS, and significant differences between the tumor and control groups, and the trauma and control groups were observed. Target proteins were isolated and purified using SPE and SDS-PAGE. Thioredoxin 1 (Trx1) was found to be differentially expressed. In the serum of children with Wilms' tumors, there was an increase in the level of the post-traumatic stress-related inflammatory factor, Trx1, as compared with the normal control group. Thus, the results of this study indicate that Trx 1 presents a potential post-traumatic stress-related factor of Wilms' tumors.
\end{abstract}

Correspondence to: Professor Jiaxiang Wang, Department of Surgery, The First Affiliated Hospital, Zhengzhou University, 1 Jianshe Road, Zhengzhou, Henan 450052, P.R. China

E-mail: wjiaxiang175@163.com

Key words: Wilms' tumors, protein marker, post-traumatic stress, proteomics, thioredoxin 1

\section{Introduction}

Wilms' tumors, also known as the embryonic tumors of the kidneys, are one of the most common malignant, solid retroperitoneal tumors observed in children, accounting for $5.5 \%$ of all solid tumors in children (1) and $>80 \%$ of genitourinary tumors in children $<15$ years of age. Although early diagnosis is important for deciding treatment methods and ensuring satisfactory outcomes, the early symptoms of Wilms' tumors are not clear. Therefore, the identification of diagnostic biomarkers is crucial.

Proteomics can rapidly detect protein expression at different stages of disease occurrence using high-throughput screening to detect specific protein-labeled molecules. These highly-expressed, specific proteins are useful in clinical practice, as they can assist in early diagnosis and may become targets for disease treatment. In previous years, a number of methods, including surface-enhanced laser desorption/ionization-time of flight mass spectrometry (SELDI-TOF-MS) and matrix-assisted laser desorption/ionization time of flight mass spectrometry (MALDI-TOF-MS) technologies $(2,3)$, have been demonstrated to successfully detect specific markers for various types of cancer, including ovarian, prostate, pancreatic, colon and breast cancer (4-8).

Post-traumatic stress disorder is complex in nature, encompassing changes in the neuroendocrine system, and in metabolic and immune function, together with altered cytokine levels, which culminate to provoke and accelerate tumor development (9). Since stress-related factors may produce proteins that interfere with the identification of specific proteins in the serum of patients with Wilms' tumors, the aim of the present study was to investigate these post-traumatic stress-related serum factors. Not only can traumatic stress-related factors be found to exclude interference for later screening and identification, the occurrence and development of tumors can also be understood from the aspect of inflammation. To analyze the effect of post-traumatic stress-related inflammatory factors on the development of Wilms' tumors and to identify putative biomarkers, SELDI-TOF-MS, MALDI-TOF-MS, solid-phase extraction (SPE), SDS-PAGE and high-performance liquid chromatography-mass spectrometry (LC-MS) were used in 
tandem to detect proteins in the sera of children with Wilms' tumors and compare them with sera isolated from children with post-traumatic stress disorder and normal children to identify post-traumatic stress-related factors. Using this approach, highly-expressed specific proteins were screened and identified in the sera of children with Wilms' tumors and those with post-traumatic stress disorder. The specific protein marker of post-traumatic stress disorder can be distinguished from the specific marker that we had obtained from the serum of patients with Wilms' tumors in our previous study (10), and may be used as a reference for excluding the interfering effects of traumatic factors.

\section{Materials and methods}

Collection and treatment of the serum samples. The study protocol was approved by the ethics review committee of Zhengzhou University (Zhengzhou, China). Written informed consent was obtained from each patient or their guardian. All serum samples used in the current study were provided by the Department of Pediatric Surgery of The First Affiliated Hospital of Zhengzhou University and included 61 pre-operative serum samples from children with Wilms' tumors, of which 28 presented with stage I tumors, 18 with stage II tumors, 12 with stage III tumors and 3 with stage IV tumors. A total of 34 serum samples were obtained from children with post-traumatic stress disorder (with sera collected within 1-3 days of injury sustained during incidents, including car accidents), and 60 serum samples were obtained from normal children who underwent routine medical examinations. Whole blood samples were harvested from the peripheral veins in the morning prior to breakfast in all participants. After $1-2 \mathrm{~h}$ at $4^{\circ} \mathrm{C}$, the samples were centrifuged for $15 \mathrm{~min}$ at $3,000 \mathrm{x} \mathrm{g}$. The sera were collected, placed in numbered tubes and preserved separately at $-80^{\circ} \mathrm{C}$.

Screening of differentially-expressed serum proteins. The serum was gradually thawed in an ice bath for 30-60 min and then centrifuged at $10,000 \mathrm{x}$ g for $5 \mathrm{~min}$ at $4^{\circ} \mathrm{C}$. Serum (5 $\mu \mathrm{l})$ was added to $10 \mu \mathrm{l}$ U9 serum solution, and was mixed together at $4^{\circ} \mathrm{C}$ on a vibrating shaker $(600 \mathrm{x}$ g; Thermo Fisher Scientific, Inc., Waltham, MA, USA) for $30 \mathrm{~min}$. The serum treated with U9 was diluted with binding buffer (PeproTech, Rocky Hill, NJ, USA) to $200 \mu \mathrm{l}$, mixed at $4^{\circ} \mathrm{C}$ on a vibrating shaker $(600 \mathrm{x} \mathrm{g})$ for $2 \mathrm{~min}$, and loaded onto a WCX2 protein chip (Ciphergen Biosystems, Fremont, CA, USA).

Firstly, the standard chip was used with proteins of known molecular weight to adjust for errors of molecular weight in the SELDI-TOF-MS system (Ciphergen Biosystems), to reach an error level of $<0.1 \%$. Next, the chip was placed together with the serum proteins into the mass spectrometer for detection. The original data were collected using Proteinchip Software version 3.2 (Ciphergen Biosystems) with the laser intensity set to 185 , the detection sensitivity set to 7 and the upper limit of detection set at 100,000 mass/charge $(\mathrm{m} / \mathrm{z})$. The range of optimized collected data was $2,000-20,000 \mathrm{~m} / \mathrm{z}$. Certain factors were excluded based on previous studies $(11,12)$. To exclude these factors, recombinant proteins were added to each group to screen for the differential peak. If the differential peak corresponded to the peak value of a certain factor to be excluded (the same $\mathrm{m} / \mathrm{z}$ ), this was screened out and no further purification and identification were performed. The differential peaks of post-traumatic specific factors were identified.

Data processing. Correction of the original data was performed using Biomarker Wizard software version 3.1 (Ciphergen Biosystems) to homogenize the total ionic strength and molecular weight. The Biomarker Wizard software and protein chip data analysis system were used to remove the noise and subtract the baseline with the discrete wavelet. The peak of each sample was obtained using a method of finding the local extreme, and peaks with signal-to-noise ratios of $>2$ were filtered out. The minimum threshold of the cluster analysis was set to $10 \%$, and the peaks with $<0.3 \% \mathrm{~m} / \mathrm{z}$ were categorized. Wilcoxon rank sum test was performed for the initially screened $\mathrm{m} / \mathrm{z}$ peaks to obtain P-values and subsequently evaluate the ability of each peak in differentiating various groups. Mass spectrometric data of each sample were further analyzed using the non-linear support vector machine (SVM) (13) to screen and identify differentially-expressed proteins. The radial basis function was used for SVM, the $\gamma$ parameter was set to 0.6 and the penalty function was set to 19 .

Statistical analysis. Subsequent to the noise being filtered, the original mass spectrometric data were treated by cluster analysis, and Student's t-tests were performed for the mass spectrometric data of each group. $\mathrm{P}<0.05$ was considered to indiciate a statistically significant difference. Differentially-expressed proteins between the groups were identified.

Isolation of serum proteins. Subsequent to the serum proteins being separated into different groups with organic phase concentrations of 30, 50, 70 and 100\% using SPE (Waters, Milford, MA, USA), they were packed in an Eppendorf ${ }^{\circledR}$ tube (Eppendorf, Hamburg, Germany), marked clearly and then divided according to hydrophilicity. Each sample was dried to $10 \mu \mathrm{l}$ in a vacuum drying device (Thermo Fisher Scientific, Inc.), subsequent to which, $5 \mu \mathrm{l}$ sample buffer was added, the samples were boiled for $15 \mathrm{~min}$ and centrifuged at $5,000 \mathrm{x} \mathrm{g}$, and the supernatant was collected. The sample was next separated by gel electrophoresis, and the gel was cut into several pieces based on the corresponding molecular weight axis, placed in different Eppendorf ${ }^{\circledR}$ tubes, washed, decolorized, dried and incubated with trypsin for $15 \mathrm{~h}$.

Identification of differentially-expressed proteins. Proteins (1.5 $\mu \mathrm{l})$ separated by SPE and electrophoresis were mixed with $1.5 \mu \mathrm{l} \alpha$-cyano-4-hydroxycinnamic acid (CHCA; Thermo Fisher Scientific, Inc.), placed on the target plate and dried. Sample calibration was performed using cytochrome $c$ (molecular weight, 12,361.96; (Thermo Fisher Scientific, Inc.) plus CHCA and insulin (molecular weight, 5,734.51; (Thermo Fisher Scientific, Inc.) plus CHCA. The target plate was placed in the MALDI-TOF-MS device (Bruker Daltonics, Billerica, MA, USA) for detection. The target protein peak was tracked, and serum samples with a protein peak $\mathrm{m} / \mathrm{z}$ of 5,816 were identified.

Identification of specific proteins. Trypsin-digested serum proteins were identified by peptide mass fingerprinting using 
Table I. Expression level of proteins with a mass/charge of 5,816 Da.

\begin{tabular}{lcc}
\hline Groups & Cases, $\mathrm{n}$ & $\begin{array}{c}\text { Mean } \\
\text { expression } \\
\pm \mathrm{SD}\end{array}$ \\
\hline Trauma & 34 & $3,674.6 \pm 255.7$ \\
Tumor & 61 & $2,128.3 \pm 137.2$ \\
Normal & 60 & $30.4 \pm 7.8$ \\
\hline
\end{tabular}

$\mathrm{SD}$, standard deviation.

LC-MS followed by Swiss-Prot database analysis using the Mascot search engine.

MS data processing and database searching. The MS data processing software used was Launchpad version 2.4 (Shimadzu, Nakagyo-ku, Kyoto, Japan). A spectrum search was performed using the Mascot search engine of mouse and human protein sequences, and the number of maximum allowable restriction enzyme cutting sites was set to 1 . The fixed modification was cysteine iodoacetyl, and the variable modification was methionine oxidation. Furthermore, the peptide mass tolerance was set to $\pm 0.3 \mathrm{Da}$.

Verification of post-traumatic stress-related protein markers using western blot analysis. Proteins were separated by SDS-PAGE and transferred to a polyvinylidene fluoride membrane. The membranes were incubated with primary polyclonal rabbit anti-human thioredoxin 1 (Trx1; cat. no. bs-0458R; 1:1,000; Beijing Biosynthesis Biotechnology Co., Ltd., Beijing, China) or GAPDH (cat. no. bs-0459R; 1:1,000; Beijing Biosynthesis Biotechnology Co., Ltd.) antibodies for $2 \mathrm{~h}$ at $37^{\circ} \mathrm{C}$. The membranes were washed three times with Tris-Buffered Saline and Tween 20 (Beijing Biosynthesis Biotechnology Co., Ltd.), followed by incubation with horseradish peroxidase conjugated-goat anti-rabbit IgG secondary antibodies (cat. no. ZDR-5306; 1:5,000; Zhongshan Golden Bridge Biotechnology Co., Ltd., Beijing, China) for $2 \mathrm{~h}$ at $37^{\circ} \mathrm{C}$. Bands were visualized using enhanced chemiluminescence followed by image development and fixing. GAPDH was used as an internal control. The negatives were scanned, and the gray values of the protein bands in each negative were measured using the ImagePro plus 6.0 software (Media Cybernetics, Bethesda, MD, USA). The gray values were divided by group, and SPSS 13.0 software (SPSS, Inc., Chicago, IL, USA) was used to compare the gray values in the different groups.

\section{Results}

SELDI-TOF-MS analysis. SELDI-TOF-MS was used to screen sera from patients in the tumor, trauma and normal control groups to identify specific proteins with an $\mathrm{m} / \mathrm{z}$ of 5,816 . Subsequent to the initial screening and statistical analysis of the mass spectra data from the tumor and normal control groups, peaks of $15 \mathrm{~m} / \mathrm{z}$ were obtained (P<0.05; Fig. 1). SVM was used to screen out models with the highest Youden's indices (i.e., the difference between the true and false positive rates) of the predictive value. Significantly higher expression of proteins with an $\mathrm{m} / \mathrm{z}$ of 5,816 was observed in the tumor group (mean, 2,128.3 \pm 137.2 ; $652.6 \pm 134.2$ in stage I tumors, $1,152.6 \pm 423.1$ in stage II tumors, 1,652.6 \pm 523.1 in stage III tumors and 2,252.6 \pm 723.1 in stage IV tumors) as compared with children in the control group $(30.4 \pm 7.8)(\mathrm{P}<0.05$; Table I). When the potential markers were taken as the input values, leave-one-out cross-validation proved that the specificity of differentiating the combination model of this marker on the test set was $100.0 \%$ and the sensitivity was $100.0 \%$. Fig. 2 shows the simulated protein electrophoresis between the tumor, normal and trauma groups. Significantly greater levels of proteins with an $\mathrm{m} / \mathrm{z}$ of 5,816 were also noted in the serum of the trauma group $(3,674.6 \pm 255.7)$ as compared with the normal group $(30.4 \pm 7.8)(\mathrm{P}<0.05$; Fig. 1; Table I).

Identification of the putative biomarker. Subsequent to the target protein being isolated and purified using SPE and SDS-PAGE, the target peptide produced by trypsin digestion was identified using high performance LC-MS (Fig. 3). A Swiss-Prot protein sequence database query via the Mascot search engine revealed that the protein with an $\mathrm{m} / \mathrm{z}$ of 5,816 may be thioredoxin 1 (Trx1). In addition, the matching rate of the detected peptide amino acid sequence and the human Trx1 sequence in the database was $46 \%$ (Table II).

Verification of the target protein was performed using western blot analysis of 30 randomly selected blood samples ( $\mathrm{n}=10$ per group) to assess Trx1 expression, which was normalized to GAPDH. For each experiment, two cases from each group were selected. As shown in Fig. 4, the highest levels of Trx 1 were observed in the trauma group $(\mathrm{P}<0.05)$, and the lowest levels were found in the normal control group $(\mathrm{P}<0.05)$. In addition, significantly higher Trx1 levels were detected in the tumor group as compared with the control group $(\mathrm{P}<0.05)$.

\section{Discussion}

In the current study, SELDI-TOF-MS was used to screen the sera of patients in the tumor, trauma and control groups. Protein with an $\mathrm{m} / \mathrm{z}$ of 5,816 was isolated and purified using SPE and SDS-PAGE, and was identified as Trx1. Finally, the presence of significantly increased Trx1 levels in the tumor group was verified by western blot analysis.

At present, Wilms' tumors cannot be cured completely, and the treatment is mainly targeted at patients with mid- to late-stage tumors, and includes surgery and chemotherapy. The main reason for the poor prognosis of patients with Wilms' tumors is the delay in early diagnosis and consequently, treatment initiation. Thus, it is critical to find a simple but accurate method for the early detection of Wilms' tumors.

Reiche et al (14) reported a positive correlation between stress and tumor development. Stress affects tumor development mostly by regulating the neuroendocrine-immune network (15). Stress-induced changes in the circadian rhythm of the body also play a significant role, and these changes are mediated by changes in endocrine mediators (e.g. glucocorticoids, thyrotrophin, growth hormone and luteinizing hormone), metabolic disorders (changes in body temperature, proteins and enzymes) and immune dysfunction, to finally induce and accelerate tumor development and escape of tumor cells from immune surveillance (9). 
Table II. Protein fragments with a mass/charge of 5,816.

\begin{tabular}{lcrr}
\hline Mass/charge & Protein & Peptide sequence & Rate of coverage, $\%$ \\
\hline 5,816 & Thioredoxin 1 & MIKPFFHSLSEKVGEFSGANKE & 46.00 \\
\hline abtained from Swiss-Prot database analysis. & & $65.00^{\mathrm{a}}$ \\
\hline
\end{tabular}
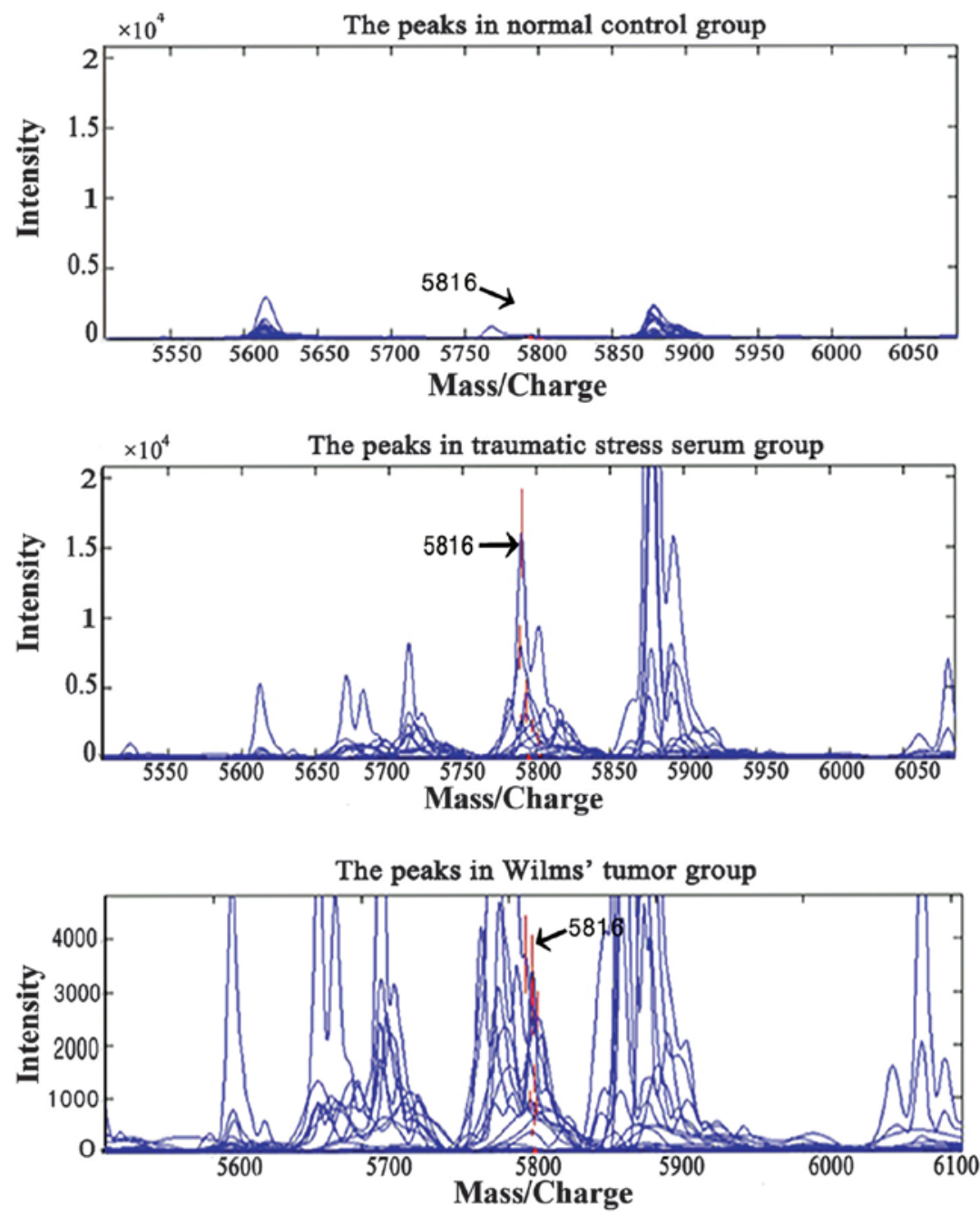

Figure 1. Spectra for proteins with an $\mathrm{m} / \mathrm{z}$ of 5,816 detected in the sera of all three groups. The red marking represents $\mathrm{m} / \mathrm{z} 5,816$ in the normal, trauma and tumor groups. $\mathrm{m} / \mathrm{z}$, mass/charge.

In children with Wilms' tumors, tumor compression and bleeding may induce the release of post-traumatic stress-related factors, thereby affecting the sensitivity and accuracy of Wilms' tumor-specific proteins. Furthermore, certain small molecular weight proteins cannot be easily detected due to their own characteristics and low concentrations. Therefore, it is important to identify a factor with a molecular weight similar to that of the factor obtained in our previous studies, which is specific to Wilms' tumors alone (10). In the present study, a protein with an $\mathrm{m} / \mathrm{z}$ of 5,816 was identified as Trx1, with a coverage of $46 \%$, suggesting that trauma-related factors exist in the serum of children with Wilms' tumors. However, their direct or indirect roles in pediatric tumor initiation or progression should be verified by further studies.
Trx1, with a molecular weight of $12 \mathrm{kDa}$, exists in almost every organism, and exhibits key functions in a number of important biological activities, including redox signaling; it is also a critical factor in tumor development (16). Specifically, Trx 1 can directly inhibit the activity of apoptosis signal-regulated kinase-1 to reduce the rate of tumor cell death and enhance tumor cell growth factor expression, thereby accelerating tumor cell growth $(17,18)$. Trx 1 can also induce the synthesis of vascular endothelial growth factor to promote angiogenesis and the extension of tumor blood vessels. In addition, it can antagonize the cancer suppressor protein in the advanced stage of cancer, promoting tumor metastasis (19). Trx1 is also overexpressed in colon cancer, significantly accelerating tumor invasion and metastasis (20). 


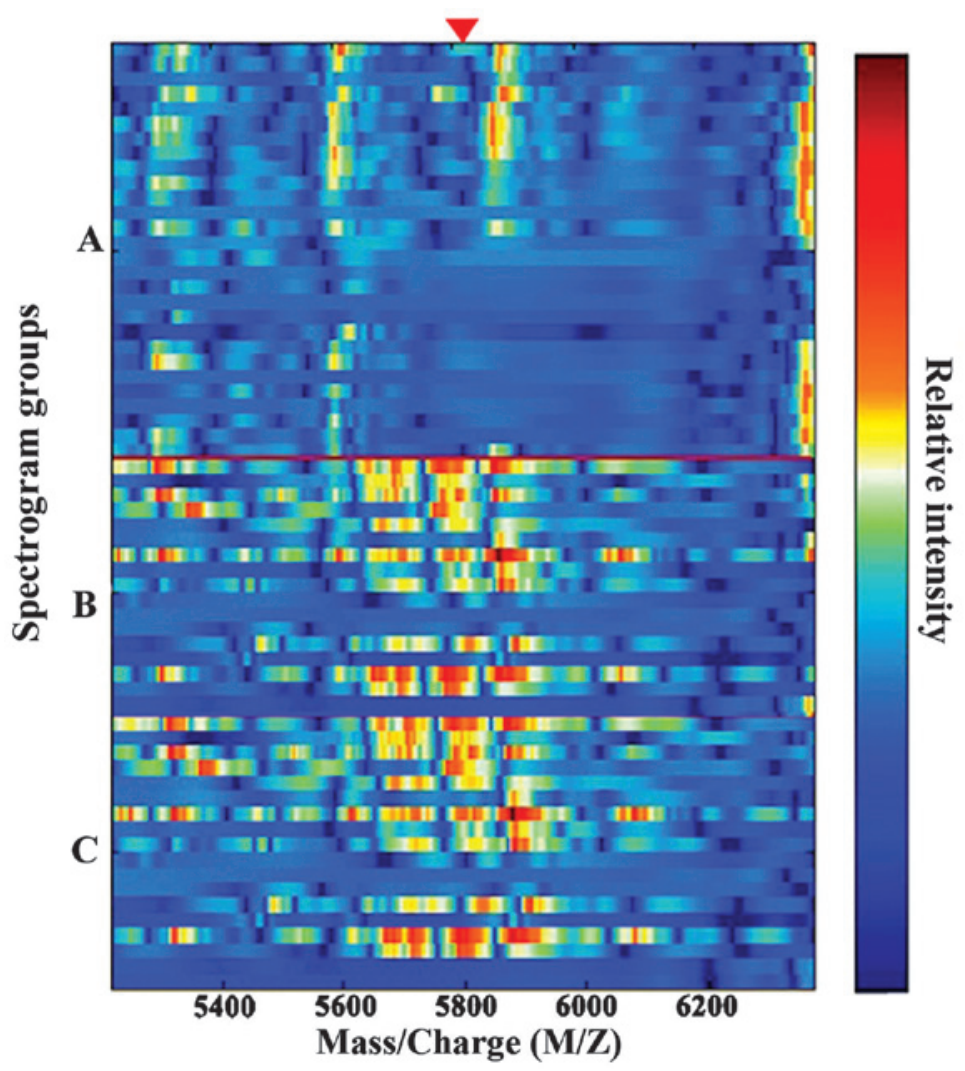

Figure 2. Simulation of electrophoresis of proteins with a mass/charge (m/z) of 5,816 (represented by the red triangle) in (A) normal, (B) tumor and (C) trauma groups.

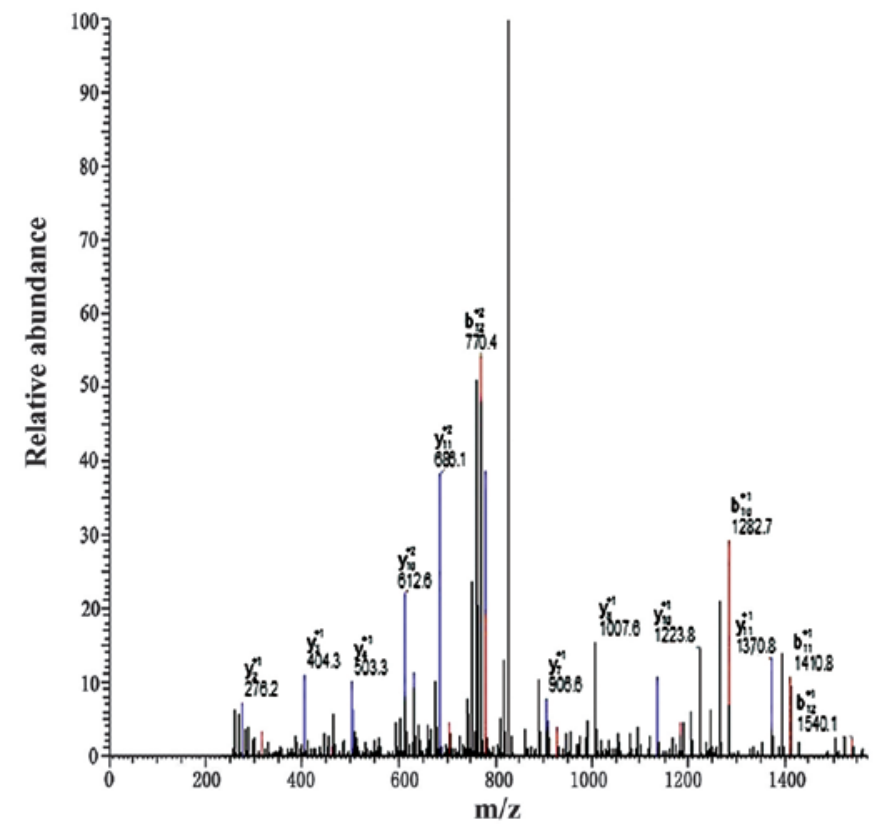

Figure 3. Mass spectra of peptides produced following trypsin digestion of the target protein with a mass/charge $(\mathrm{m} / \mathrm{z})$ of 5,816 Da.

The current study applied SELDI-TOF-MS technology along with SVM to screen out post-traumatic stress-related protein markers with an $\mathrm{m} / \mathrm{z}$ of 5,816 . The specificity and sensitivity of the identified marker, Trx1, were each $100 \%$, and the intensity of its expression may be associated with factors such as the individual differences in the serum

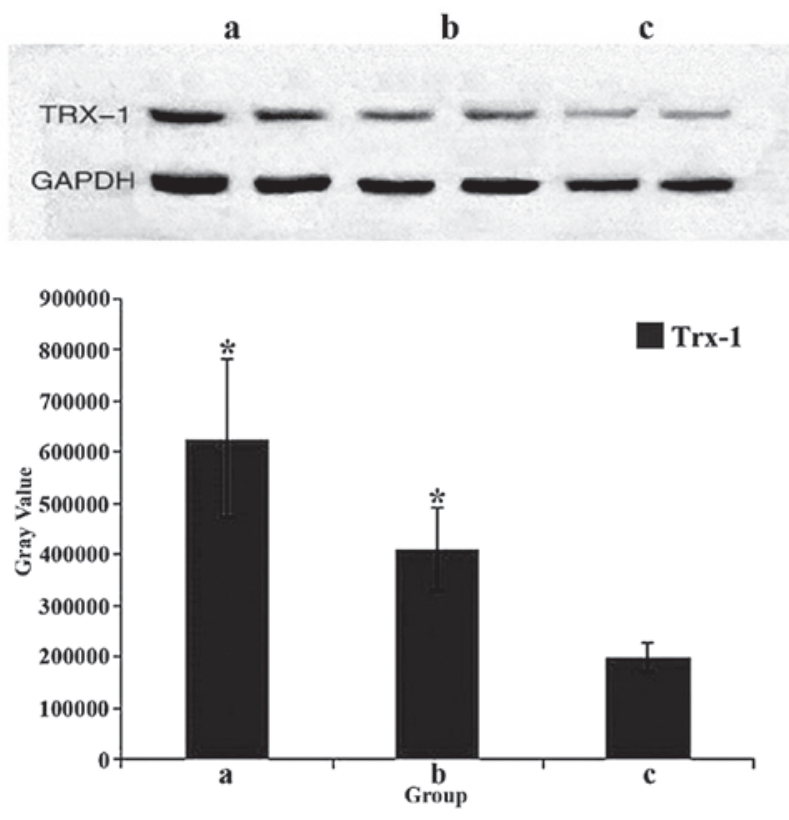

Figure 4. Thioredoxin $1(\operatorname{Trx} 1)$ protein levels were determined by western blot analysis and normalized to GAPDH in the trauma, tumor and control groups. ${ }^{*} \mathrm{P}<0.05$. a, trauma group; b, tumor group; c, normal group.

samples, experiment process and errors. Together with inflammatory markers and those biomarkers described in previous studies (e.g. serum amyloid A and apolipoprotein CIII) $(21,22)$, Trx1 forms an ideal diagnostic marker, as its levels were significantly higher in the sera of children with Wilms' tumor and in those patients with post-traumatic stress disorder in the present 
study. The relatively high expression level in the serum of children with tumors is in accordance with its high expression level in adult tumors, which suggests that it is a post-traumatic stress-related serum factor of Wilms' tumors. Combined with the results of our previous study (10), the current study demonstrated that the post-traumatic stress-related specific protein that was screened out was Trx1. The post-traumatic stress-related factor Trx1 was expressed in the serum of children with Wilms' tumors. The results shown in this study present the novel idea that enzymes indicative of oxidative stress may be used as markers for the serological diagnosis, malignancy grading and prognostic evaluation of Wilms' tumor, and may form the basis of future studies with larger cohorts.

\section{Acknowledgements}

This study was supported by the National Natural Science Foundation of China (grant no. 81071782).

\section{References}

1. Kaatsch P: Epidemiology of childhood cancer. Cancer Treat Rev 36: 277-285, 2010.

2. Maurya P, Meleady P, Dowling P and Clynes M: Proteomic approaches for serum biomarker discovery in cancer. Anticancer Res 27: 1247-1255, 2007.

3. Diamandis EP: Mass spectrometry as a diagnostic and a cancer biomarker discovery tool: opportunities and potential limitations. Mol Cell Proteomics 3: 367-378, 2004.

4. Wang J, Zhang X, Ge X, Guo H, et al: Proteomic studies of early-stage and advanced ovarian cancer patients. Gynecol Oncol 111: 111-119, 2008.

5. Skytt A, Thysell E, Stattin P, Stenman UH, Antti H and Wikstrom P: SELDI-TOF MS versus prostate specific antigen analysis of prospective plasma samples in a nested case-control study of prostate cancer. Int J Cancer 121: 615-620, 2007.

6. Liu D, Cao L, Yu J, et al: Diagnosis of pancreatic adenocarcinoma using protein chip technology. Pancreatology 9: 127-135, 2009.

7. Hundt S, Haug U and Brenner H: Blood markers for early detection of colorectal cancer: a systematic review. Cancer Epidemiol Biomarkers Prev 16: 1935-1953, 2007.
8. Gonçalves A, Bertucci F, Birnbaum D and Borg JP: Proteic profiling SELDI-TOF and breast cancer: clinical potential applications. Med Sci (Paris) 23: 23-26, 2007 (In French).

9. Cao WG: Mechanism of tumor development affecting traumatic stress. Yi Xue Zong Shu 6: 830-832, 2012 (In Chinese).

10. Wang J, Wang L, Zhang D, et al: Identification of potential serum biomarkers for Wilms tumor after excluding confounding effects of common systemic inflammatory factors. Mol Biol Rep 39: 5095-5104, 2012.

11. Chechlinska M, Kowalewska M and Nowak R: Systemic inflammation as a confounding factor in cancer biomarker discovery and validation. Nat Rev Cancer 10: 2-3, 2010.

12. Hou JM, Zhao X, Tian L, et al: Immunotherapy of tumors with recombinant adenovirus encoding macrophage inflammatory protein 3 beta induces tumor-specific immune response in immunocompetent tumor-bearing mice. Acta Pharmacol Sin 30: 355-363, 2009.

13. Liu Y: Active learning with support vector machine applied to gene expression data for cancer classification. Chem Inf Comput Sci 44: 1936-1941, 2004.

14. Reiche EM, Nunes SO and Morimto HK: Stress, depression, the immune system and cancer. Lancet Oncol 5: 617-625, 2004.

15. Shastri A, Bonifati DM and Kishore U: Innate immunity and neuroinflammation. Mediators Inflamm 2013: 342931, 2013.

16. Kodama A, Watanabe H, Tanaka R, et al: A human serum albumin-thioredoxin fusion protein prevents experimental contrast-induced nephropathy. Kidney Int 83: 446-454, 2013.

17. Navakoski de Oliveira K, Andermark V, von Grafenstein S, et al: Butyltin (IV) benzoates: inhibition of thioredoxin reductase, tumor cell growth inhibition and interactions with proteins. Chem Med Chem 8: 256-264, 2013.

18. Lu A, Wangpu X, Han D, et al: TXNDC9 expression in colorectal cancer cells and its influence on colorectal cancer prognosis. Cancer Invest 30: 721-726, 2012.

19. Kakolyris S, Giatromanolaki A, Koukourakis M, et a1: Thioredoxin expression is associated with lymph node status and prognosis in early operable non-small cell lung cancer. Clin Cancer Res 7: 3087-3091, 2001.

20. Lincoln DT, Alyatama F, Mohammed FM, et al: Thioredoxin and thioredoxin reductase expression in thyroid cancer depends on tumour aggressiveness. Anticancer Res 30: 767-775, 2010.

21. Zhang J, Guo F, Wang L, Zhao W, Zhang D, Yang H, Yu J, Niu L, Yang F, Zheng S and Wang J: Identification of apolipoprotein C-I as a potential Wilms' tumor marker after excluding inflammatory factors. Int J Mol Sci 15: 16186-16195, 2014.

22. Zhang Q, Wang J, Dong R, Yang S and Zheng S: Identification of novel serum biomarkers in child nephroblastoma using proteomics technology. Mol Biol Rep 38: 631-638, 2011. 